Signal \& Image Processing : An International Journal (SIPIJ) Vol.6, No.5, October 2015

\title{
A REVIEW OF COMPUTER VISION SYSTEM FOR THE VEHICLE IDENTIFICATION AND CLASSIFICATION FROM ONLINE AND OFFLINE VIDEOS
}

\author{
Baljit Singh Mokha ${ }^{1}$ and Satish Kumar ${ }^{2}$ \\ ${ }^{1}$ Department of Computer Science and Applications, \\ Panjab university, Chandigarh (India) \\ ${ }^{2}$ Department of Computer Science and Applications, \\ Panjab University, SSG Regional Centre , Hoshiarpur, Punjab (India)
}

\begin{abstract}
The traffic on the roads is increasing day by day. There is dire need of developing an automation system that can effectively manage and control the traffic on roads. The traffic data of multiple vehicle types on roads is also important for taking various decisions related to traffic. A video based traffic data collection system for multiple vehicle types is helpful for monitoring vehicles under homogenous and heterogeneous traffic conditions. In this paper, we have studied different methods for the identification, classification and counting vehicles from online and offline videos in India as well as other countries. The paper also discusses the various applications of video based automatic traffic control system. The various challenges faced by the researchers for developing such systems are also discussed.
\end{abstract}

\section{KEYWORD}

Vehicle Identification, Vehicle Classification, Vehicle Counting, Traffic Control.

\section{INTRODUCTION}

Computer vision is an important field of artificial intelligence where decision about real world scene having high dimensional data is taken. The general steps used in this process are acquiring, processing and analyzing the image and convert it into numerical or symbolic form. It is used to understand the scene electronically and the process is equivalent to the ability of human vision. The numerical or symbolic information of a scene is decided based on the appropriate model constructed with the support of object geometry, physics, statistic, and learning theory. The scene under consideration is converted into the image(s) or the video(s), comprising of many images, using camera(s) focused from different locations on a scene. The various vision related areas such as seen reconstruction, event detection, video tracking, object recognition, object pose estimation and image restoration are considered as subareas of computer vision. Similarly, various other fields such as image processing, image analysis and machine vision are also closely related to 
Signal \& Image Processing : An International Journal (SIPIJ) Vol.6, No.5, October 2015

computer vision. The techniques and applications of various above said areas overlap with each other. Moreover, the techniques used in all these areas are more or less identical. The difference in names only lies on the applications where the techniques are applied.

Image processing and image analysis both deals with $2 \mathrm{D}$ images. In image processing an image is transformed into another by applying some operations such as contrast enhancement, edge detection, noise removal and geometrical transformations. The image contents are not interpreted in image processing whereas in computer vision the interpretation of images is made based on the properties of the contents they contain. Computer vision may include analysis of 3D images from 2D.

\section{VIDEO ANALYSIS / VIDEO INTERPRETATION}

Video analysis is the process in which a video is automatically analyzed to detect and determine the temporal and spatial events. In video analysis some algorithms are implemented as software on general machine or as hardware in video processing units. In video analysis the video motion detection, video tracking, background abstraction, behaviour analysis and situation awareness are the main factors.

Video interpretation is a video telecommunication service that uses devices such as video cameras or videophones to provide sign language or spoken language interpreting language. This is done through the remote or offsite interpreter, in order to communicate with whom there is a communication barrier. Video interpreter facilitates communication between the participants who are located together at the other site. They are communicating by using headphones or microphone.

Object identification is a process where identity of an object under consideration in a scene is made. It may include the identification of an individual, animal, bird, vehicle, tree, river etc. The given scene is converted into an image using image capturing device and some pre-processing techniques are applied on it to convert it to desire form. In case of content based interpretation smaller regions of interests (ROI) are extracted from images using simple and fast computing techniques. These ROI are further analysed by more computationally demanding techniques to produce a correct interpretation.

\subsection{Vehicle identification and classification system (VICS)}

The VICS system for identification and classification of moving vehicles on the road side from the videos is a great importance today. In India the traffic related information are gathered manually. One of the easy way to exchange information related to traffic between different computers by using network which is helpful for making many kind of decision related to traffic management. A VICS system can identify and classify vehicles on the basis of road side videos. AVICS system is helpful for traffic control and collecting statistics data related to vehicles which are helpful for taking many decision. A number of vehicle identification and classification systems have been developed by various prominent authors but $100 \%$ accuracy is not available. In VICS system, vehicle identification and classification can be done in two ways online and offline. In offline system, the identification and classification of vehicles is done from the videos related to the traffic whereas in online system, the images are captured by CCTV camera installed on the road side and the system identifies and classifies the vehicles directly from that video(s). 
Signal \& Image Processing : An International Journal (SIPIJ) Vol.6, No.5, October 2015

Vehicle identification and classification system (VICS) is an intelligent vehicle recognition system used to manage traffic on roads. There is dire need of monitoring and controlling traffic on road using efficient and effective cost effective method. In VICS the images of the vehicles are captured using a video camera installed on road side. In VICS the decision may be made on the basis of a single camera on one side or multiple cameras installed at different locations at particular angle depending upon the requirement and level of sophistication of the system. The video are converted into shots and frames, then features extraction and classification method are applied to identify and classify the vehicles. General steps in video based vehicle identification and classification system, and associated applications are given in Figure 1.

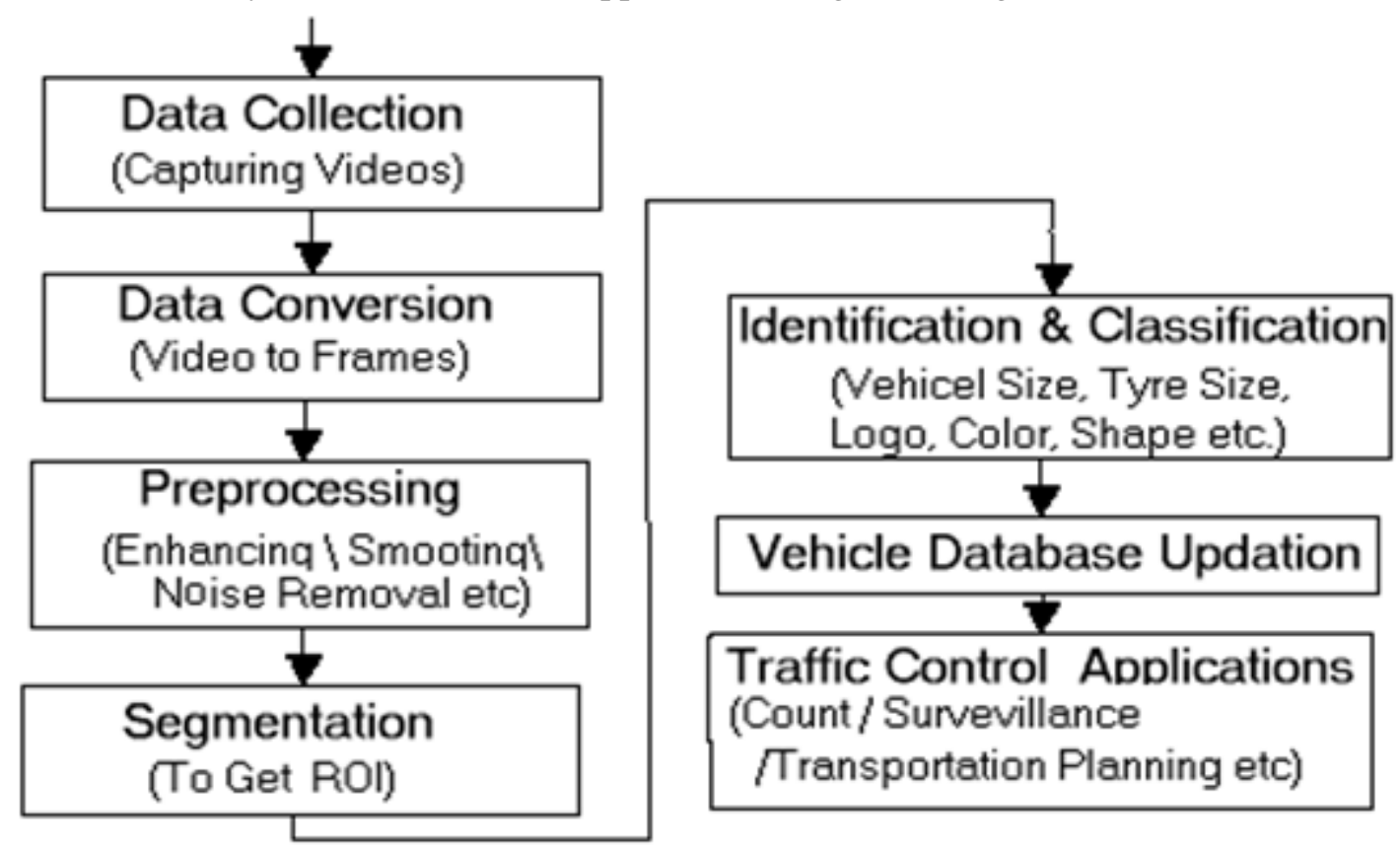

Figure 1: General steps in Video based identification and classification system (VICS).

The traffic on roads can be broadly classified into two categories i.e. homogenous and heterogeneous. Homogeneous traffic is a hypothetical synchronized flow of traffic of similar vehicles where all vehicle move with the same speed, irrespective of time and maintain the same space gap between them. The condition under which the traffic move is also called as homogenous traffic conditions. Heterogeneous traffic is unsynchronized and unregulated i.e. there is an irregular movement of all type of vehicles. In VICS, the traffic data extractor is used to detect and track each object moving through the scene to check its speed, path, class and counting. It analysis the video sequences in order to locate the object passed through a view of the cameras and to track them until they leave the obscured seen. The object parameter extractor analyse each object, as soon as it is available, in the object database list already stored in system. It identifies, classifies and tracks that object and all its parameters are saved the result list.

\section{LITRATURE REVIEW}

Vehicle identification and classification system, Lai et al [1], used to calculate the speed of the vehicles where a number of loops are automatically assigned to each lane. To automatic calculate the speed of a vehicle the inductive loop detector method is applied. The merit of doing this is 
Signal \& Image Processing : An International Journal (SIPIJ) Vol.6, No.5, October 2015

that a) It accommodates pan-tilt-zoom (PTZ) actions without the further requirement of human interaction. b) The size of the virtual loops is much smaller for estimation of accuracy. This enables the use of standard block-based motion estimation techniques that are well developed for video coding. c) The number of virtual loops per lane is large. The motion content of each block may be weighted and the collective result offers a more reliable and robust approach in motion estimation. There is no failure rate associated with the virtual loops or physical installation. As the loops are defined on the image sequence, changing the detection configuration or redeploying the loops to other locations on the same image sequence requires only a change of the assignment parameters. d) Virtual loops may be reallocated anywhere on the frame, giving flexibility in detecting different parameters.

Dhanya et al [2] developed a computer vision system for detecting and tracking the moving vehicle at day time and night time. First the videos are converted into frames and background and foreground of the image are detected. The headlight and the taillight of the vehicle is used for detecting and identifying the vehicle, after that image segmentation and pattern analysis techniques are applied. A fast bright object is identified and classified spatial clustering.

Mishra et al [3] develop an algorithm for detection and classification of vehicle in heterogeneous traffic. The entire process is divided into four steps i.e. camera calibration, vehicle detection, speed estimation, and classification. Vehicle detection is carried using background subtraction and blob tracking methods. Speed of the vehicle is estimated by using start and stop lane marker and calibration parameter. Classification of vehicles depends upon the various features of the detected vehicles. These features give the input to SVM for classification. A non-linear kernel is used as the classifier.

Chaoyang et al [4] recognizes logos in video stream in real-time. A new technique is developed that combines both coarse template matching approach and pair wise learning method together. The logo recognition becomes effective and efficient by eliminating the false alarms and further refines the recognition results. Image alignment for template matching improves the stability of the coarse stage. Experimental results show that this approach outperform the DOT matching approach and traditional multiple classifiers combination.

Daigavane et al [5] developed an application based on neural network for vehicle detection and classification. This system identifies and classifies the vehicles with their success rate $90 \%$. Vehicle are tracked by using blob tracking method and neural networks classify these vehicles on the basis of length and height There have been cases where the system is unable to do the classification correctly. When multiple vehicles move together, with approximately the same velocity, they tend to get grouped together as one vehicle. Also, the presence of shadows can cause the system to classify vehicles incorrectly.

Chen et al [6] investigate the effectiveness of state-of-the-art classification algorithms to categorise road vehicles for an urban traffic monitoring system using a multi-shape descriptor. The analysis is applied to monocular video acquired from a static pole-mounted road side CCTV camera on a busy street. These are used to classify the objects into four main vehicle categories i.e. car, van, bus and motorcycle. Image analysis for vehicle classification can be generally categorised into three principle approaches: model-based classification, Feature based classification and Measurement based classification. A number of experiments have been conducted to compare support vector machines (SVM) and random forests (RF) classifiers. 10- 
Signal \& Image Processing : An International Journal (SIPIJ) Vol.6, No.5, October 2015

fold cross validation has been used to evaluate the performance of the classification methods. The results demonstrate that all methods achieve a recognition rate above $95 \%$ on the dataset, with SVM consistently outperforming RF. A combination of MBF and IPHOG features give the best performance of $99.78 \%$.

Iwaski et al [7] studied road traffic flow surveillance under various environmental circumstances that cause poor visibility of vehicles on road. Authors used thermal images taken with infrared thermal cameras to detection vehicle. Two methods have been proposed. The first method uses pattern recognition for windshields and their surroundings to detect vehicles. The second method uses tires' thermal energy reflection areas on a road as the detection targets.

$\mathrm{M}$ et al [8] developed a system SCOCA, for counting and classifying vehicles automatically. The aim is to collect data for statistical purpose. The traffic data are extractor by installing CCTV cameras on a pool. After detecting the scene, the second step is object parameter extractor. The methodology used for tracking an object is model based, region based, contour based and feature based. The object attributes determined are class, speed and path. The model based classification is used. The SCOCA system works in real time at 25 frames per second. A separate test has been conductor to measure the performance of second label cycle, motorcycle classifier based on SVM (Support Vector Machine) classifier techniques (189 vehicles, 45 bicycles, 144 motorcycles extracted from two video sequences. The classifier provides an average error rate $6.7 \%$.

Deb [9] developed an automatic driver assistance system to alert a driver about driving environment. The most common approach used to vehicle detection is active sensor such as radar based system, laird ("Light Detection and Ranging") and acoustic based. Radar based system can see 150 meter ahead in fog or rain. To find the location of vehicles, the three approaches are used i.e. knowledge based, stereo based and motion based. The vehicle identification are done on the basis of symmetry, colour, shadow, corners, vertical and horizontal edges, texture, and vehicle light. In stereo vision system, the three methods used are disparity map, inverse perspective mapping and motion based and the location are find by using template based and appearance based.

Betke et al [10] described a real-time vision system that analyzes colour videos taken from a forward-looking video camera in a car driving on a highway. The system is a combination of colour, edge, and motion information to recognize and track the road boundaries, lane markings and other vehicles on the road. Cars are recognized by matching templates that are cropped from the input data online and by detecting highway scene features and evaluating the way they relate to each other. Cars are also detected by temporal differencing and by tracking motion parameters that are typical for cars. The system recognizes and tracks road boundaries and lane markings using a recursive least-squares filter. Experimental results demonstrate robust, real-time car detection and tracking over thousands of image frames. The data includes video taken under difficult visibility conditions.

Changlasetty et al [11] presented a system for identification and classification of vehicles where vehicles are tracked by using width, length and parameter area extracted using image techniques. Traffic data are recorded by stationary camera. Background of the image are extracted by using mixture model. For the classification LABVIEW and neural networks is trained to classify vehicles using data monitoring WEKA tools It is used in intelligent transport system for Indian 
Signal \& Image Processing : An International Journal (SIPIJ) Vol.6, No.5, October 2015

cites. A feed-forward neural network is trained to classify vehicles using data mining WEKA toolbox.

Chung et al [12] developed a real-time vision-based vehicle detection system that employs an online boosting algorithm. It is an online AdaBoost approach that cascades various strong classifiers instead of a single strong classifier. The cascade of strong classifiers for vehicle detection is online updated in response to traffic changing environment. The online algorithms efficiently catch the parameter based on the incoming image and their performance is up to date. This approach has been successfully validated in real traffic environments by performing experiments with an onboard charge-coupled-device camera in a roadway vehicle.

Shaoqing et al [13] real-time classification method is proposed for heavy traffic flow multi-lanes roads, which can classify vehicles into cars, trucks and buses. In this system three cameras are installed at 60 degree they focus on the lanes and vehicle features are extracted from it. The presence of car is determined by the colour of LPR and the absence of the car by segmented the image by combination of position mapping function. The feature extraction methods hybrid insensitive noise edge detection method based on Sobel operator and colors, the second is regions mergence according to colors and positions are applied to determine the car. Lastly noncars are classified into trucks and buses by a fuzzy rules classifier.

Suryatali et al [14] presents a system where a camera captures the images of the vehicles passing through a toll booth thus a vehicle is detected through camera. The classification of vehicles is done on the basis of area of vehicles. This information is further passed to the 'Raspberry Pi' which is having a web server set up on it. The Raspberry Pi is a credit card-sized single-board computer developed in the UK. The job of Raspberry pi in this system is processing large quantities of data and also it will keep detailed log of vehicles which are in the system.The Raspberry $\mathrm{Pi}$ is a good choice for a webserver that will not receive too much traffic and only uses around 5 Watts of power. This system can also counts moving vehicles from pre-recorded videos or stored videos by using the same algorithm. A new toll collection system is low cost alternative among all other system. This system is based on computer vision vehicle detection using open CV library embedded in Linux platform.

Xuehua et al [15] proposes an algorithm which can solve the problems effectively by the improved Gaussian mixture model and Support vector machine. First of all, the paper introduce an improved Gaussian mixture model which can effectively detect the moving objects and resolve the problems of Gaussian mixture model sensitive to light changes. Then the paper designs some classifiers to recognize the pedestrians and vehicles by the idea of the improved SVM. The experimental results show that the method has a high recognition rate and can also satisfy the real-time intelligent transportation surveillance

Mohammed et al [16] presents an offline system that is capable to detect the moving object and classify it into classes. The classifications are made on the basis of the situation and dynamic feature of the moving objects. All sequential features are arranged to improve the classification significantly. Three approaches are used for detecting the moving objects temporal differences, Background Subtraction Algorithms (BSA) and optical flow. An algorithm which is capable to detect and classify moving objects given a sequence of frames acquired by a static camera is developed. Features of the moving object is obtained from the Background Subtraction method 
Signal \& Image Processing : An International Journal (SIPIJ) Vol.6, No.5, October 2015

then processed to enhance both the static and dynamic features of the target. The classification algorithm makes use of these features vector which are sequentially arranged.

Brendan et al (2006) [17] presents a system that recognizes the car with 3 different features namely license plate, logo and colour. The spatial segmentation and the temporal segmentation yields the moving objects. Object in video are tracked and detected using particle filter. The particle filter is a Bayesian sequential importance sampling technique. It consists of two essential steps: prediction and update. The paper analyzes particle filter for tracking the object. The approach can further be combined with the training model developed using features for detecting and tracking cars in real time. This is shown on a real-time system monitoring highway traffic. The system retrieves online video at $10 \mathrm{frames} / \mathrm{sec}$ and conducts tracking and classification simultaneously and Results are improved $74 \%$ to $88 \%$.

Jin-Hua et al (2010) [18] present a system that detects the types of vehicles to achieve the identification of vehicles. The extracted image heaving some noise due to that gives the misjudgement of the vehicle. In this situation Naive Bayesian classification is introduced. While characteristic parameter errors occurred, the probability values of different types of vehicles are calculated by Naive Bayesian classification, and compare the probability values.

Zhan et al [19] presents a novel fine-grained vehicle recognition system that utilizes the collaborative feedback scheme for detection-classification-tracking in surveillance video. The researcher takes data 200cars heaving 3 category and some negative samples. The Harr-like feature are extracted from the image offline and SVM are used as a classifier for interclass variation. The detector localizes the motion objects in every frame except that have been observed and tracked, and the best matching object with the given initial car is identified by the classifier and be tracked in the subsequent frames until it is not present. The experiment result shows that this approach can effectively locate the best matching car from the frames when the target car appears.

Cui et al [20] propose a novel method based on semantic component analysis and scale consideration. The foreground of the image is estimated with the GMM and the candidate component are extracted with the DPM(deformable part based model) model. To extract the physical components of the vehicle using the DPM and to learn the structure of the components using the latent SVM. The model is trained in a fully supervised framework in which the positive samples are annotated with bounding boxes, and the parameters representing the layout of the parts are learned as latent information.

Rijurekha et al [21] present a comprehensive study of all available ITS systems, including both research prototypes and deployed systems. They also discussed some open research problem related ITS in India and also discuss some public and private organization that play a role in Indian traffic management. Sensor technology is used for ITS system. There are several existing modes of sensing: static sensing, where sensors are statically placed on the road, mobile sensing, where sensors are placed in the moving vehicles and hybrid sensing, where both in-vehicle and on-road infrastructure are needed.

Mallikarjuna et al [22] present an offline image processing system named TRAZER to collect data under mixed traffic conditions. TRAZER is capable of tracking vehicles even under highly congested traffic conditions. This system can capture vehicle from no lane system and track the 
Signal \& Image Processing : An International Journal (SIPIJ) Vol.6, No.5, October 2015

small size vehicle. TRAZER could also capture acceleration/deceleration behaviour of vehicles. Among the macroscopic characteristics, classified traffic volume, average occupancy, and average speeds are collected. Some of microscopic and macroscopic traffic feature are extracted.

Vaddi et al (2015) [23]devolved a system to identify unauthorized vehicles on roads as a part of vehicle surveillance and traffic data analysis useful in decision making in terms of safety evaluation, pavement design, funding, forecasting, and modelling. Vehicles chassis are constructed in a significantly greater variety of geometries as compared to other objects. There are number of dissimilarities in vehicles like height, number of wheels, body shape and colour. Three important categories in this area are Wavelet based, methods based on contour or edge and feature extraction with classification. Applied wavelet analysis and learn a Support Vector Machine (SVM) or neural network (NN) to recognize cars on highways.

Ponnu et al(2013)[24] aims to study the traffic flow in a three-lane two-way undivided road in the city of Chennai through developing multi-class speed-flow relationships using both linear and Bureau of Public Roads (BPR) models. It has been found from the study that models with total volume perform the same irrespective of units used being vehicles or PCU and models with classwise (with and without direction) consistently perform better than total volume models. It was also found that linear models work better than other models for all vehicle classes modelling 2wheeler speeds and two-wheelers, cars and LCVs have the greatest influence on the speeds of almost all the category of vehicles and the stream. One of the other important conclusions from the study is that an optimum use of class-wise and $\&$ direction-wise models can offer models of better fit than two individual class and class-direction models.

Rahane et al (2014)[25] presents an algorithm for on-road vehicle detection problem using cascade of boosted classifiers. This approach filters different non-target (negative) samples in different stages of cascaded structure according to their level of similarity with target object class. Then compared the performance of local and global texture features in combination with boosted Haar like features. The best performance for on-road obstacle detection is achieved by Adaboost with Haar-like feature along with SVM and histograms of oriented gradients (HOG) features. The results reveal that the presented approach improves the detection efficiency.

Chhoriya et al (2013)[26] presents a system that would pay the toll automatically and reduce the queue at the toll booth. The system capture the no plate and convert it into a text using ANPR tool pay from the customer account and then open the gate. Moreover, if a vehicle is stolen and an entry is being made in the central database by the police then if the vehicle passes through the toll booth then silent alarm would buzz which would indicate the operator at the toll booth that the vehicle is a stolen vehicle.

Chitaliya et al(2012)[27] designed a vehicle classifier using the discrete Curvelet transform to increase the efficiency of classifier, 3 class structures designed with respect to the ratio of length and width of the vehicle or person on the road. Each image is pre-processed with un-sharp filtering which provides edge and convert it into a binary image. Binary image are converted into discrete curve let heaving low frequency and high frequency. Feature vector matrixes are drawn and Eigen value is calculated. The experiments carried out on different types of vehicle images. The results of the classifier show the efficiency to handle the 
Signal \& Image Processing : An International Journal (SIPIJ) Vol.6, No.5, October 2015

real time dataset. The details of work done for vehicle identification and classification system, online or offline videos, in foreign and India are given in Table1.

Table 1: Summarization of studies on vehicle identification and classification vehicles.

\begin{tabular}{|c|c|c|c|c|}
\hline Method & Identification/Classification & Foreign/Indian & Success rate & References \\
\hline $\begin{array}{c}\text { SVM to identify } \\
\text { PPlive, } \\
\text { PPstream, UUsee, } \\
\text { QQlive and } \\
\text { Sopcast }\end{array}$ & $\begin{array}{l}\text { Classification } \\
\text { Online video }\end{array}$ & China & $93.5 \%$ & $\begin{array}{l}\text { Liu et.al } \\
(2004)\end{array}$ \\
\hline $\begin{array}{l}\text { Window based } \\
\text { online learning } \\
\text { algorithm }\end{array}$ & $\begin{array}{l}\text { Identification } \\
\text { Offline videos }\end{array}$ & Canada & $80 \%$ & $\begin{array}{l}V \text { Nair et.al } \\
(2005)\end{array}$ \\
\hline $\begin{array}{l}\text { Algorithm based } \\
\text { on Width to } \\
\text { Height Ratio } \\
\text { (WHR)and } \\
\text { Base to Abdomen } \\
\text { Ratio (BAR) }\end{array}$ & Identification & Malaysia & $93 \%$ & S Mohammed et.al (2005) \\
\hline Bayesian Network & $\begin{array}{l}\text { Classification and identification } \\
\text { online }\end{array}$ & $\begin{array}{l}\text { California } \\
\text { USA }\end{array}$ & $88 \%$ & B.M et al (2006) \\
\hline $\begin{array}{l}\text { online boosting } \\
\text { algorithm }\end{array}$ & $\begin{array}{l}\text { Classifier and detecaion } \\
\text { Offline and offline }\end{array}$ & Foreign & $97 \%$ & $\begin{array}{l}\text { W Chung et.al } \\
\quad(2010)\end{array}$ \\
\hline$M B F$ and IPHOG & $\begin{array}{l}\text { Classification } \\
\text { Online Videos }\end{array}$ & London U.K & $99.78 \%$ & Z Chen et al (2011) \\
\hline $\begin{array}{l}\text { virtual detection } \\
\text { loops }\end{array}$ & $\begin{array}{c}\text { Counting and classification } \\
\text { online }\end{array}$ & USA & $97.4 \%$ & $\begin{array}{l}\text { SLiet.al } \\
\text { (2013) }\end{array}$ \\
\hline $\begin{array}{l}\text { thermal energy } \\
\text { in traffic flow } \\
\text { surveillance }\end{array}$ & $\begin{array}{c}\text { Thermal } \\
\text { Vehicle detection }\end{array}$ & Japan & $91.2 \%$ & S Shantaiya et.al(2014) \\
\hline $\begin{array}{l}\text { Fine-grained } \\
\text { Recognition } \\
\text { algorishms and } \\
\text { SVM } \\
\text { Only for cars }\end{array}$ & $\begin{array}{l}\text { Classifier } \\
\text { Offline videos }\end{array}$ & China & $97.38 \%$ & J Zhan et.al( 2014) \\
\hline$H M M$ & Identification and classification & USA & $866 \%$ & $\begin{array}{c}\text { A. Jazayeri et.al } \\
(2015)\end{array}$ \\
\hline$S V M$ & Classification online & London & $-6.7 \%$ & $M$ et al \\
\hline $\begin{array}{c}D P M+S L R \\
+C S D(D S C)\end{array}$ & $\begin{array}{l}\text { Back view/ } \\
\text { Front view }\end{array}$ & China & $\begin{array}{l}0.9933 \% \\
0.9964 \%\end{array}$ & G Cui etal \\
\hline $\begin{array}{c}G M M+D P M+ \\
C S D(G D C)\end{array}$ & $\begin{array}{l}\text { Back viewd } \\
\text { Front view }\end{array}$ & China & $\begin{array}{l}0.8841 \% / \\
0.9286 \%\end{array}$ & G Cui et.al \\
\hline $\begin{array}{c}G M M+D P M+ \\
S L R+C S D \\
(G D S C) \\
D P M+S R C \\
(D P M S)\end{array}$ & $\begin{array}{l}\text { Back view' } \\
\text { Front view } \\
\text { Back view' } \\
\text { Front view }\end{array}$ & $\begin{array}{l}\text { China } \\
\text { China }\end{array}$ & $\begin{array}{l}0.9286 \% \\
0.9796 \% \\
0.9796 \% / \\
0.9987 \%\end{array}$ & $\begin{array}{l}\text { G Cui et.al } \\
\text { G Cui et.al }\end{array}$ \\
\hline $\begin{array}{l}\text { Algorithms } \\
\text { Gaussian } \\
\text { distribution } \\
\text { models }\end{array}$ & $\begin{array}{l}\text { Both for identification and classification } \\
\text { of vehicle and pedestrians }\end{array}$ & China & $\begin{array}{l}91.4 \% \text { for } \\
\text { Pedestrian } \\
93.75 \% \text { for } \\
\text { Vehicles }\end{array}$ & $X$ Song et. al \\
\hline $\begin{array}{c}\text { Scale invariant } \\
\text { feature transform } \\
\text { (SIFT) }\end{array}$ & Vehicle Recognitation & India & $89.5 \%$ & D Belongie, 2005 \\
\hline
\end{tabular}


Signal \& Image Processing : An International Journal (SIPIJ) Vol.6, No.5, October 2015

\begin{tabular}{|c|c|c|c|c|}
\hline TRAZER & Classification offline & Guwahati & $8825 \%$ & $\begin{array}{l}\text { C. Mallikarjuna } \\
\text { et.al(2009) }\end{array}$ \\
\hline $\begin{array}{c}\text { Neural } \\
\text { Network/Blob } \\
\text { tracking }\end{array}$ & Identification and classification online & Wardha, India & $90 \%$ & P.Met al (2011) \\
\hline $\begin{array}{l}\text { Quantized } \\
\text { wavelet } \\
\text { features } \\
\text { method } \\
\text { SVM }\end{array}$ & Detection online & $\begin{array}{l}\text { New Delhi } \\
\text { India }\end{array}$ & $93.94 \%$ & $\begin{array}{l}\text { S Kumar et.al } \\
\quad(2012)\end{array}$ \\
\hline $\begin{array}{l}\text { Random forest } \\
\text { algorithm. }\end{array}$ & Classification online & $\begin{array}{l}\text { New Delhi } \\
\text { India }\end{array}$ & $94.8 \%$ & $\begin{array}{l}\text { S Kumar et.al } \\
\quad(2012)\end{array}$ \\
\hline $\begin{array}{c}\text { adaptive } \\
\text { background } \\
\text { modelling and } \\
\text { classification is } \\
\text { achieved by novel }\end{array}$ & $\begin{array}{l}\text { Identification and classification } \\
\text { Online }\end{array}$ & Mumbai & $828 \%$ & $\begin{array}{l}\text { PKumar et.al } \\
\quad(2013)\end{array}$ \\
\hline $\begin{array}{l}\text { Decision tree } \\
\text { Algorithms }\end{array}$ & $\begin{array}{l}\text { Classification } \\
\text { online }\end{array}$ & $\begin{array}{l}\text { Ahmadabad } \\
\text { India }\end{array}$ & $85 \%$ & S Shantaiya et.al(2014) \\
\hline SVM RBF & In both & $\begin{array}{l}\text { Vijayawada } \\
\text { India }\end{array}$ & $90.00 \%$ & RS Vaddi et.al( 2015) \\
\hline $\begin{array}{c}\text { Traffic } \\
\text { Analyzer and } \\
\text { Enumerator- } \\
\text { TRAZER. }\end{array}$ & $\begin{array}{l}\text { Vehicle detection } \\
\text { offline }\end{array}$ & $\begin{array}{l}\text { Guwahati } \\
\text { India }\end{array}$ & $95 \%$ & $\begin{array}{l}\text { C. Mallikarjuna et.al } \\
\qquad(2015)\end{array}$ \\
\hline $\begin{array}{c}\text { knowledge-guided } \\
\text { boundary } \\
\text { algorithms }\end{array}$ & $\begin{array}{l}\text { template matching for automatic vehicle } \\
\text { identification }\end{array}$ & $\begin{array}{l}\text { Uttar Pradesh } \\
\quad \text { India }\end{array}$ & $95 \%$ & RKumar et.al \\
\hline $\begin{array}{l}\text { Random forest } \\
\text { algorithm. }\end{array}$ & $\begin{array}{c}\text { Online } \\
\text { Classifier }\end{array}$ & Madras & $95 \%$ & $S M$ et.al \\
\hline
\end{tabular}

\section{APPLICATIONS OF VICS}

The various applications of image/video based image analysis, recognition and understanding are as $[4,6,10,14,18,23,27]$

1. Video Surveillance.

2. Traffic Management System.

3. Vision based intelligent Transport System.

4. Intersection Control.

5. Incident Detection

6. Vehicle Classification.

7. Monitoring.

8. Revenue collection.

9. Historical Traffic Data.

10. Congestion Map and travel time estimate.

11. Public Transport information.

12. Individual Vehicle Management.

13. Accident Handling.

14. Conventional Driver Assistance system.

15. Traffic Surveillance. 
Signal \& Image Processing : An International Journal (SIPIJ) Vol.6, No.5, October 2015

16. Transportation Planning and Intelligent Traffic Guidance system.

17. Vehicle counting.

18. License plate localization.

19. Tool collection system.

\section{Challenges}

While designing system based on image/video based image analysis, recognition and side traffic management, a number of challenges are faced by the researcher and some of these are as:

- In western countries like USA, lane marking system is implemented. Vehicles are allowed to move in specific rows according to speed limit and vehicle type, etc. But in India in most of the cases traffic system is non lane based.

- Road conditions are more varied and traffic is unstructured, there is lack of discipline and overloaded vehicle movement is quite natural.

- In India, vehicles are parked frequently by the sides of the roads. There is no separate system for vehicle parking management.

- Roads are not only occupied with vehicles, so many obstacles on roads create disturbance to the traffic. Pedestrians do not have separate ways for their movement in most of the cases.

- Shapes of the vehicles have a key role in recognition; there is high intra-class variance among Indian vehicles. It creates the chances for miss recognition.

- Within same vehicle class there are large variety and models. These look different in size and appearance. It is generally observed in Indian vehicles like cars and Truck's.

- Vehicles detection using optical sensor is very challenging due to huge within class variability in vehicles appearance.

- Hundred of images and billion of classes.

\section{CONCLUSiOnS}

The comprehensive details of the vehicle identification and classification system from the online and offline videos are present. The summery of this work is as follows:

1. The majority of work has been carried out for offline videos.

2. The majority of the work has been done in foreign countries such as China, Canada, Malaysia, California USA, London U.K, and Japan. The interesting part of such research is that there is proper lane system and rule and regulations on roads. The vehicles are moving according to their speed. 
Signal \& Image Processing : An International Journal (SIPIJ) Vol.6, No.5, October 2015

3. The various systems developed vehicle management do not give $100 \%$ accuracy.

4. All the real time systems developed in developed courtiers are only operating under certain Environmental constraints.

5. The various techniques used are as:

a) Pre-processing: Background Subtraction Algorithm (BSA), Kalman filter algorithm, HSV colour model theory, signal-to-noise (SNR),

b) Feature extraction: Haar-like features, HOG feature extraction, Gaussian background model, Blob tracking method

c) classification techniques Width to Height Ratio (WHR), SVM, Fine-grained Recognition, MBF and IPHOG, PHOG, AdaBoost approach, Naive Bayesian classification, SIFT(Scale invariant Feature Transform), standard NearestNeighbour (NN) classifier, Gaussian mixture model, LDA (Linear Discriminant Analysis), discrete cosine transform, Gabor transform, discrete wavelet transform, Inductive Loop Detectors, Traffic Analyzer and EnumeratorTRAZER, hidden Markov model, Optical flow direction intensity, Global Nearest Neighbour (GNN), SCOCA- System for Counting and Classifying Automatically vehicles, Decision tree, A histogram based nonlinear kernel, LABVIEW, WEKA

6. Work done in Indian context is purely for mixed traffic conditions. They are lacking in within same vehicle class there are large varieties and models. Shapes of the vehicles have a key role in recognition, there is high intra-class variance among Indian vehicles Roads are not only occupied with vehicles, so many obstacles on roads create disturbance to the traffic.

\section{REFERENCES}

[1] H. S. Lai and H. C. Yung, "Vehicle-Type Identification Through Automated Virtual Loop Assignment and Block-Based Direction-Biased Motion Estimation", IEEE Transactions on Intelligent Transportation System, Vol. 1, No. 2, June 2000.

[2] K. Dhanya, M. Manimekalai, B.Asmin and G. Vani, "Tracking and Identification of Multiple Vehicles ".

[3] P. Mishra, M. Athiq, A. Nandoriya and S. Chaudhuri,"Video based Vehicle Detection and Classification in Heterogeneous Traffic Conditions using a Novel Kernel Classifier" IETE journal of research vol 2013.

[4] C. Zhao, J. Wang, C. Xie and H. Lu," A COARSE-TO-FINE LOGO RECOGNITION METHOD IN VIDEO STREAMS" National Laboratory of Pattern Recognition, CASIA, Beijing China

[5] P.M. Daigavane and M.B. Daigavane," Vehicle Detection and Neural Network Application for Vehicle Classification" International Conference on Computational Intelligence and Communication Systems 2011.

[6] Z. Chen and T. Ellis," Multi-shape Descriptor Vehicle Classification for Urban Traffic" International Conference on Digital Image Computing: Techniques and Applications 2011.

[7] Y. Iwasaki, M. Misumi, and T Nakamiya, "Robust Vehicle Detection under Various Environments to Realize Road Traffic Flow Surveillance Using An Infrared Thermal Camera" The Scientific World Journal, Volume 2015, Article ID 947272, Hindawi Publishing Corporation. 
Signal \& Image Processing : An International Journal (SIPIJ) Vol.6, No.5, October 2015

[8] S. Messelodi, M. Modena, and M. Zanin "'A computer vision system for the detection and classification of vehicles at urban road intersections" Springer-Verlag 2005 London Limited.

[9] K. Deb and K. Nathr "Vehicle Detection Based on Video for Traffic Surveillance on road". Int. J Comp Sci. Emerging Tech Vol-3 No 4 August, 2012.

[10] M. Betke, E. Haritaoglu and S. Davis, "Real-time multiple vehicle detection and tracking from a moving vehicle" Machine Vision and Applications pp 69-83Springer-Verlag 2000.

[11] B. Changalasetty, A. Badawy, W. Ghribi, H. Ashwi, A. Mohammed, A.Shehri, S. Thota and R. Medisetty, "Identification and Classification of Moving Vehicles on Road" Computer Engineering and Intelligent Systems pp 2222-2863 Vol.4, No.8, 2013.

[12] W.Chang and W.Cho , "Online Boosting for Vehicle Detection" IEEE TRANSACTIONS ON SYSTEMS, MAN, AND CYBERNETICS VOL. 40, NO. 3, JUNE2010

[13] M.Shaoqing and L.Zhengguang, "Real-time Vehicle Classification Method for Multilane Roads "ICIEA 2009.

[14] A. Suryatali and V.B. Dharmadhikari, "Computer Vision Based Vehicle Detection for Toll Collection System Using Embedded Linux". International Conference on Circuit, Power and Computing Technologies 2015

[15] X. Song, L. Wang, H. Wang and Y. Zhang, "Detection and identification in the Intelligent Traffic Video Monitoring System for pedestrians and vehicles".

[16] M. Osman and A.Bakar, "Classification of Moving Objects Using Active Vision Computer Vision" Computer Vision, Video, and Image Processing Laboratory(CVVIP) IEEE 2005.

[17] S. Kumar and N.Sivanandam," Object Detection and Tracking in Video using particle filter "ICCCNT'12 IEEE-2012 Coimbatore, India.

[18] T. Jin-hua and Jiangsu," Research of Vehicle Video Image Recognition Technology Based on Naive Bayesian Classification" Third International Conference on Information and Computing 2010.

[19] J. Zhan, H. Zhang and X. Luo," Fine-grained Vehicle Recognition via Detection-ClassificationTracking in Surveillance Video" IEEE International Conference on Digital Home2014.

[20] G. Cui, Q. Wang and Y. Yuan," Vehicle Detection Based on Semantic Component Analysis "Chinese Academy of Sciences China.

[21] R. Sen and B. Raman," Intelligent Transport Systems for Indian Cities".

[22] C. Mallikarjuna, A. Phanindr and R. Rao "Traffic Data Collection under Mixed Traffic Conditions Using Video Image Processing" Journal of transportation engineering 2009.

[23] S. Vaddi, P.Boggavarapu, K. Anne and V. Siddhartha "Computer Vision based Vehicle Recognition on Indian Roads" International Journal of Computer Vision and Signal Processing, 2015.

[24] B. Ponnu, J. Thomas, K. Jha , G.Asaithambi and K. Srinivasan, "Vehicle Class-wise Speed Volume model for Three-lane Undivided Urban Roads" Social and Behavioral science 104 pp468-476 2013.

[25] K. Radhe and U. Saharkar, "Technique identification for road traffic congection solution in Talegaon Dabhade state highway-55" JOURNAL OF INFORMATION, KNOWLEDGE AND RESEARCH Vol-3 pp30975 - 6744 OCT 2014.

[26] P. Chhoriya, G.Paliwal and P. Badha, "Image Processing Based Automatic Toll Booth in Indian Conditions" International Journal of Emerging Technology and Advanced Engineering vol-3 April 2013.

[27] G.Chitaliya and I.Trivedi, "Automated Vehicle Identification System based on Discrete Curvelet Transform for Visual Surveillance and Traffic Monitoring System" International Journal of Computer Applications Vol-57no.1, November 2012. 
Signal \& Image Processing : An International Journal (SIPIJ) Vol.6, No.5, October 2015

\section{AUTHORS}

Baljit singh mokha is research scholar in Computer Science and Applications in Panjab University, Chandigarh (India).

Dr. Satish Kumar is Associate Professor in Computer Science and Applications in Panjab University, Chandigarh (India), currently posted at Panjab University SSG Regional Centre, Hoshiarpur (Punjab) and has more than fifteen years experience of teaching Post-graduate classes. He is actively engaged in research activities and guiding PhD students. He has contributed more than 25 research papers in national and international conferencesljournals repute. His areas of interest are Image Processing, Pattern Recognition and Artificial Intelligence involved with these fields.
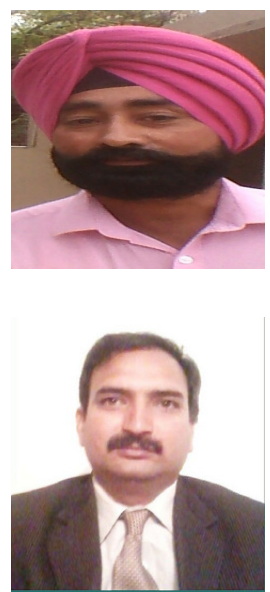